\title{
Radiation-induced brain injury: retrospective analysis of twelve pathologically proven cases
}

\author{
Dong-Soo Lee, MD¹, Mina Yu, MD¹, Hong-Seok Jang, MD¹, Yeon-Sil Kim, MD¹, \\ Byung-Ock Choi, MD', Young-Nam Kang, PhD', Youn-Soo Lee, MD², \\ Dong-Chul Kim, MD², Yong-Kil Hong, MD³ , Sin-Soo Jeun, MD³ Sei-Chul Yoon, MD ${ }^{1}$ \\ Departments of ${ }^{1}$ Radiation Oncology, ${ }^{2}$ Pathology, ${ }^{3}$ Neurosurgery, The Cancer Center of Seoul St. Mary's Hospital, \\ The Catholic University of Korea, Seoul, Korea
}

\begin{abstract}
Purpose: This study was designed to determine the influencing factors and clinical course of pathologically proven cases of radiation-induced brain injury (RIBI).

Materials and Methods: The pathologic records of twelve patients were reviewed; these patients underwent surgery following radiotherapy due to disease progression found by follow-up imaging. However, they were finally diagnosed with RIBI. All patients had been treated with 3-dimensional conventional fractionated radiotherapy and/or radiosurgery for primary or metastatic brain tumors with or without chemotherapy. The histological distribution was as follows: two falx meningioma, six glioblastoma multiform (GBM), two anaplastic oligodendroglioma, one low grade oligodendroglioma, and one small cell lung cancer with brain metastasis.

Results: Radiation necrosis was noted in eight patients and the remaining four were diagnosed with radiation change. Gender $(p=0.061)$ and biologically equivalent dose $(B E D)_{3}(p=0.084)$ were the only marginally influencing factors of radiation necrosis. Median time to RIBI was 7.3 months (range, 0.5 to 61 months). Three prolonged survivors with GBM were observed. In the subgroup analysis of high grade gliomas, RIBI that developed $<6$ months after radiotherapy was associated with inferior overall survival rates compared to cases of RIBI that occurred $\geq 6$ months $(p=0.085)$.

Conclusion: Our study demonstrated that RIBI could occur in early periods after conventional fractionated brain radiotherapy within normal tolerable dose ranges. Studies with a larger number of patients are required to identify the strong influencing factors for RIBI development.
\end{abstract}

Keywords: Radiation necrosis, Radiation injury, Chemoradiation, Brain tumor

\section{Introduction}

Radiation-induced brain injury (RIBI) is a serious complication which is commonly associated with significant functional morbidity and decreased quality of life in patients treated with brain radiotherapy. The brain is one of the most commonly irradiated sites for treating primary central nervous system (CNS) tumors as well as the palliation of metastatic tumors. Given the fact that higher radiation doses to the tumor can increase local tumor control and improve overall prognosis, radiation dose schemes have changed over the last few decades. Radiation dose escalation [1-4] and various altered

Received 24 March 2011, Revised 8 June 2011, Accepted 15 June 2011.

Correspondence: Sei-Chul Yoon, MD, Department of Radiation Oncology, Seoul St. Mary's Hospital, 505 Banpo-dong, Seocho-gu, Seoul 137-701, Korea. Tel: +82-2-2258-1526, Fax: +82-2-2258-1532, E-mail: scyoon@catholic.ac.kr

(c) This is an Open Access article distributed under the terms of the Creative Commons Attribution Non-Commercial License (http://creativecommons.org/ licenses/by-nc/3.0/) which permits unrestricted non-commercial use, distribution, and reproduction in any medium, provided the original work is properly cited.

www.e-roj.org 
fractionation schedules $[3,5]$ such as stereotactic radiosurgery [2], hypofractionated radiotherapy [3] and boost techniques $[2,4]$ are the most widely used dose schemes in recent years. With these changes, increased damage to the parenchyma of normal brain has become one of the inevitable consequences. Moreover, it is very important to differentiate radiationinduced brain necrosis (RIBN) from primary tumor progression for proper patient care management because RIBN mimics the recurrence of brain tumors in imaging studies. Although new diagnostic tools have been developed, making differential diagnoses between tumor recurrence and RIBN is still difficult.

In 1991, Emami et al. [6] reported normal organ tissue tolerance doses in various organs and tissues. These data were applied to a partial dose-volume model, and the TD 5/5 (total radiation dose that can induce $5 \%$ of complication within 5 years) of whole brain, 2/3 of brain, and 1/3 of brain were reported as 4,500 cGy, 5,000 cGy, and 6,000 cGy, respectively. More recently, the Quantitative Analysis of Normal Tissue Effects in the Clinic review [7] refined the normal tissue dosevolume tolerance guidelines for the modern three-dimensional era.

Many potential causes of RIBI have been proposed [8-12]. Generally, most cases of RIBI are known to be associated with a combination of initial vascular endothelial damage, glial cell damage, alteration of cytokine expression, and chronic inflammatory responses. A number of reports have examined cases of RIBI. However, the results were sometimes reported as diagnostic imaging changes only, and there are few data for histopathologically confirmed cases. We designed this study to determine which factors are associated with the development of RIBI. We focused on identifying potential risk factors and examining the clinical course of RIBI in patients treated with 3-dimensional (3D) conventional fractionated radiotherapy or radiosurgery.

\section{Materials and Methods}

\section{Patient population and tissue pathology}

From June 1996 to July 2006, we experienced 12 cases of surgically proven RIBI at the Department of Radiation Oncology, the Cancer Center of Seoul St. Mary's Hospital. Eleven patients had received definitive or adjuvant radiation therapy for primary CNS tumors; one patient was treated for a metastatic brain tumor from small cell lung cancer (SCLC) as a part of palliative care. All of 12 patients underwent salvage surgery because disease progression was observed in follow-up imaging studies after initial radiotherapy. All patients except two had presented specific neurologic symptoms including a number of motor or sensory changes depending on the tumor locations. These symptoms included headache, nausea, seizures, homonymous hemianopsia, aphasia, blurred vision, dysarthria, one-sided motor weakness, and/or hemiparesis.

Computed tomography and magnetic resonance imaging (MRI) results of all 12 patients were available. Brain thallium single photon emission computed tomography was performed in one glioblastoma multiform (GBM) patient, and magnetic resonance spectroscopy (MRS) was selectively performed in two patients with anaplastic oligodendroglioma and one patient with low grade oligodendroglioma. MRI results of all patients showed progressive space-occupying mass lesions and ring-like enhancement with or without peritumoral edema. MRS findings revealed malignant tumor recurrence in one anaplastic oligodendroglioma patient, and an absence of malignancies in the remaining two patients.

Pathologic specimens were reviewed by specialized neuropathologists. RIBI was defined as RIBN or radiation-induced brain change (RIBC) without evidence of viable tumor cells. RIBN was defined as a formation of coagulation necrosis combined with fibrinoid necrosis of blood vessels and hyalinization of the vascular walls within previously irradiated areas along with newly developed gadoliniumenhancing lesions on images. Patients diagnosed with nonspecific radiation changes with viable tumor cells (indicative of recurrent disease) or who did not undergo pathologic confirmation were excluded from this study.

\section{Radiation therapy and chemotherapy}

Radiation therapy was delivered with $6 \mathrm{MV}$ or $10 \mathrm{MV}$ of photon beams by a linear accerelator or CyberKnife ${ }^{\circledR}$ (Accuray, Sunnyvale, CA, USA) radiosurgery equipment. Target volumes of $3 D$ conventional fractionated treatment were as follows. The gross tumor volume (GTV) was defined as the preoperative primary tumor area with or without peritumoral edema. The clinical target volume (CTV) was defined as the GTV plus a 3D expansion of $1.5-2 \mathrm{~cm}$ for high grade tumors and $1-1.5 \mathrm{~cm}$ for low grade tumors. The CTV was slightly modified depending on the surrounding normal structures and anatomical location. The planning target volume (PTV) was defined as the CTV plus a 3D expansion of $0.3-0.5 \mathrm{~cm}$. The entire PTV was encompassed within the 90-95\% isodose lines. Field size reduction (cone down) was conducted after administering 36-45 Gy depending on the tumor characteristics. A total radiation dose of 54-60 
Gy was prescribed for the GTV according to the primary tumor histology. For one patient treated with the CyberKnife ${ }^{\circledR}$, the GTV was defined as the recurrent gross mass, and PTV was defined as GTV plus a 3D expansion of 1-2 $\mathrm{mm}$. The prescribed radiation dose was delivered to appropriately cover the PTV with adequate isodose lines.

Two meningioma patients received a single dose of 30 Gy or 24 Gy using linac-based radiosurgery. Another nine patients were treated with conventional fractionated adjuvant radiotherapy. One patient with brain metastasis from SCLC received brain radiotherapy three times. Initially, he received 24 Gy of prophylactic cranial irradiation in 12 fractions followed by 24 Gy single CyberKnife ${ }^{\circledR}$ radiosurgery for the metastatic lesions. Finally, 24 Gy of whole brain radiotherapy was administered in 12 fractions over a period of 22 months. Chemotherapy was concurrently administered to eight patients. 1-(4-amino-2-methyl-5-pyrimidinyl) methyl-3(2-chloroethyl)-3-nitrosourea hydrochloride (ACNU) was used to treat one GBM, procarbazine, CCNU, vincristine (PCV) for four GBM and two anaplastic oligodendroglioma, and temozolomide was given to one patient with low grade oligodendroglioma.

\section{Statistical analysis}

All statistical analyses were carried out using SPSS ver. 12.0 (SPSS Inc., Chicago, IL, USA). Fisher's exact test and the Mann-
Whitney $U$ test were used to compare the distribution of variables. The time between initial diagnosis or brain injury to death or the last follow-up was estimated using the KaplanMeier method. A log-rank test was used to compare the survival curves. All of the statistical tests were two-sided, and a $p<0.05$ was considered statistically significant.

\section{Results}

\section{Patient characteristics}

Baseline patient characteristics according to the disease group are listed in Table 1.

The median age was 48 years (range, 25 to 65 years). The distribution of initial diagnoses was as follows: two meningioma, six GBM (WHO grade IV), two anaplastic oligodendroglioma (WHO grade III), one low grade oligodendroglioma (WHO grade II), and one SCLC with brain metastasis. Among these, 11 patients underwent curative brain surgery followed by adjuvant radiotherapy. Median total radiation dose in the entire patient group was 57.6 Gy (range, 24 to $59.4 \mathrm{~Gy})$. When the total radiation dose was converted into a biologically equivalent dose (BED) using an $\alpha / \beta$ ratio of 3 based on a linear-quadratic model, $\mathrm{BED}_{3}$ ranged from 86.4 to 330 Gy (median, 95.04 Gy).

Table 1. Baseline patient, tumor, and treatment characteristics

\begin{tabular}{|c|c|c|c|c|c|c|c|c|}
\hline Patient & Diagnosis & Gender & Age (yr) & Tumor size $(\mathrm{cm})$ & Tumor location & $\begin{array}{l}\text { Extent of } \\
\text { surgery }\end{array}$ & $\begin{array}{c}\text { Total radiation } \\
\text { dose/Fx/days }\end{array}$ & Chemotherapy \\
\hline 1 & GBM & $M$ & 25 & $5 \times 5 \times 4.8$ & Left $P$ & GTR & $5,580 / 31 / 47$ & ACNU \\
\hline 2 & GBM & $\mathrm{F}$ & 43 & $6.5 \times 5 \times 4.3$ & Right P-0 & GTR & $5,940 / 33 / 54$ & PCV \\
\hline 3 & GBM & $F$ & 53 & $5.8 \times 5.2 \times 4.8$ & Right P & STR & $5,940 / 33 / 69$ & PCV \\
\hline 4 & GBM & $M$ & 57 & $5.8 \times 4.7 \times 4.1$ & Right F & GTR & $5,940 / 33 / 50$ & PCV \\
\hline 5 & GBM & $M$ & 59 & $4.3 \times 3.1 \times 2.8$ & Left F & STR & $5,940 / 33 / 45$ & None \\
\hline 6 & GBM & $M$ & 61 & $4 \times 2.4 \times 2$ & Left P-T & STR & $5,940 / 33 / 52$ & PCV \\
\hline 7 & Anaplastic ODG & $\mathrm{F}$ & 28 & $3 \times 2.5 \times 2$ & Right F-P & GTR & $5,940 / 33 / 46$ & PCV \\
\hline 8 & Anaplastic ODG & $F$ & 39 & $7 \times 5 \times 3$ & Right F & STR & $5,580 / 31 / 44$ & PCV \\
\hline 9 & Low grade ODG & $M$ & 39 & $4 \times 3.6 \times 3.1$ & Right F & GTR & $5,400 / 30 / 42$ & Temozolomide \\
\hline 10 & Meningioma & $F$ & 56 & $7.2 \times 7.2 \times 6$ & P-parasagital & STR & $3,000 / 1 / 1$ & None \\
\hline 11 & Meningioma & $\mathrm{F}$ & 65 & $2.6 \times 2 \times 1.8$ & F-parasagital & None & $2,400 / 1 / 1$ & None \\
\hline 12 & $\mathrm{SCLC}$ & M & 42 & $2.4 \times 2.2 \times 2.1$ & Right F & None & $\begin{array}{c}2,400 / 12 \\
2,400 / 1 \\
2,400 / 12\end{array}$ & None \\
\hline
\end{tabular}

Fx, fractions; GBM, glioblastoma multiform; P, parietal; GTR, gross tumor removal; ACNU, 1-(4-amino-2-methyl-5-pyrimidinyl) methyl3-(2-chloroethyl)-3-nitrosourea hydrochloride; P-0, parieto-occipital; PCV, procarbazine, CCNU, vincristine; F, frontal; STR, subtotal tumor removal; P-T, parieto-temporal; ODG, oligodendroglioma; F-P, fronto-parietal; SCLC, small cell lung cancer. 


\section{Pathologic assessment}

Detailed pathologic finding, specific past history, and treatment outcomes for the 12 patients are summarized in Table 2. Among the 12 patients, seven had a history of other diseases. This included five cases of diabetes mellitus, four cases of hypertension, one case of hyperlipidemia, one cerebellar infarction, one inferior wall myocardial infarction, and one arterio-venous malformation. Four patients had a combination of at least two diseases. The median time from the end of radiotherapy to the progression found by follow-up imaging was 7.3 months (range, 0.5 to 61 months). Pathologic review of surgically removed specimens found RIBN in eight patients. The median time for RIBN development in these eight cases was 4.2 months (range, 0.5 to 39.6 months). The remaining four patients were diagnosed with RIBC characterized by radiation-induced atypism, reactive gliosis, fibrin deposition, and fibrinoid degeneration without evidence of viable tumor cells. The median time for RIBC development was 13.4 months (range, 5.7 to 61 months). Serial imaging and histologic finding of RIBN in two patients are shown in Figs. 1 and 2 , respectively.

\section{Influencing factors on the radiation-induced brain injury} We conducted Fisher's exact test and Mann-Whitney $U$ test

Table 2. Detailed characteristics of patient outcomes

\begin{tabular}{|c|c|c|c|c|c|c|}
\hline Patient & $\begin{array}{c}\text { Total } \mathrm{BED}_{3} \\
\text { (Gy) }\end{array}$ & $\begin{array}{l}\text { Pathologic } \\
\text { finding }\end{array}$ & Other disease & $\begin{array}{l}\text { Elapsed time to } \\
\text { brain injury (mo) }\end{array}$ & $\begin{array}{l}\text { OS from initial } \\
\text { diagnosis (mo) }\end{array}$ & $\begin{array}{l}\text { Survival } \\
\text { status }\end{array}$ \\
\hline 1 & 89.3 & $\mathrm{C}$ & AVM & 61.0 & 84.6 & Death \\
\hline 2 & 95.0 & $\mathrm{~N}$ & Non-specific & 1.2 & 6.9 & Death \\
\hline 3 & 95.0 & $\mathrm{~N}$ & DM, HLD & 8.9 & 135.6 & Alive \\
\hline 4 & 95.0 & C & DM, HTN, Cbll infarction, MI & 10.7 & 24.1 & Death \\
\hline 5 & 95.0 & $\mathrm{~N}$ & Non-specific & 0.5 & 11.9 & Death \\
\hline 6 & 95.0 & C & DM, HTN & 5.7 & 16.8 & Death \\
\hline 7 & 95.0 & $\mathrm{~N}$ & Non-specific & 1.8 & 34.9 & Death \\
\hline 8 & 89.3 & $N$ & Non-specific & 4.4 & 49.9 & Death \\
\hline 9 & 86.4 & C & HTN & 16.0 & 91.1 & Alive \\
\hline 10 & 330 & $\mathrm{~N}$ & Non-specific & 39.6 & 218.2 & Alive \\
\hline 11 & 216 & $\mathrm{~N}$ & DM, HTN & 11.3 & 97.4 & Alive \\
\hline 12 & 296 & $N$ & $\mathrm{DM}$ & 4.0 & 38.2 & Death \\
\hline
\end{tabular}

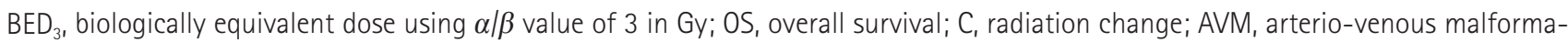
tion; $\mathrm{N}$, radiation necrosis; DM, diabetes mellitus; HLD, hyperlipidemia; HTN, hypertension; Cbll, cerebellar; MI, myocardial infarction.
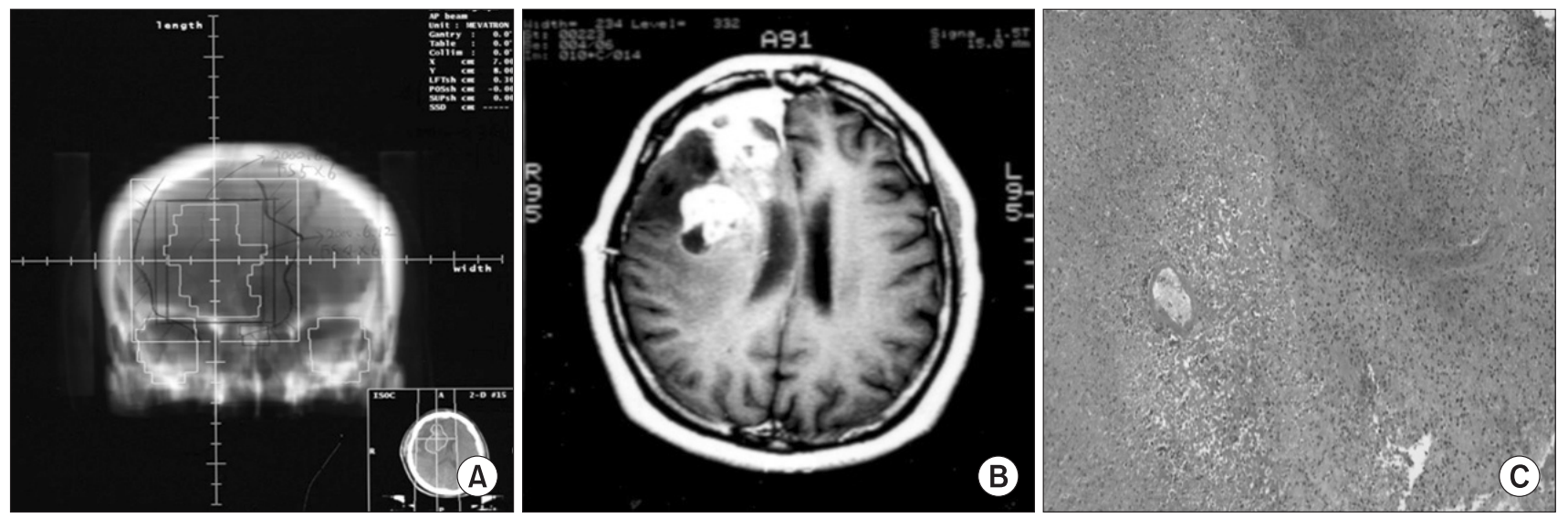

Fig. 1. A 39-year-old female (patient number 8) diagnosed with anaplastic oligodendroglioma in the right frontal lobe following subtotal tumor resection and concurrent procarbazine, CCNU, vincristine (PCV) chemoradiation with up to 55.8 Gy in 31 fractions over 44 days. The digitally reconstructed radiograph image (A), and newly developed heterogeneously gadolinium-enhancing mass with midline shifting on the T1-weighted MR image taken 4.4 months after radiotherapy (B) are shown. She underwent re-operation, and histologic findings showed radiation necrosis with the sparing of a blood vessel (C) (HEEE, X100). 

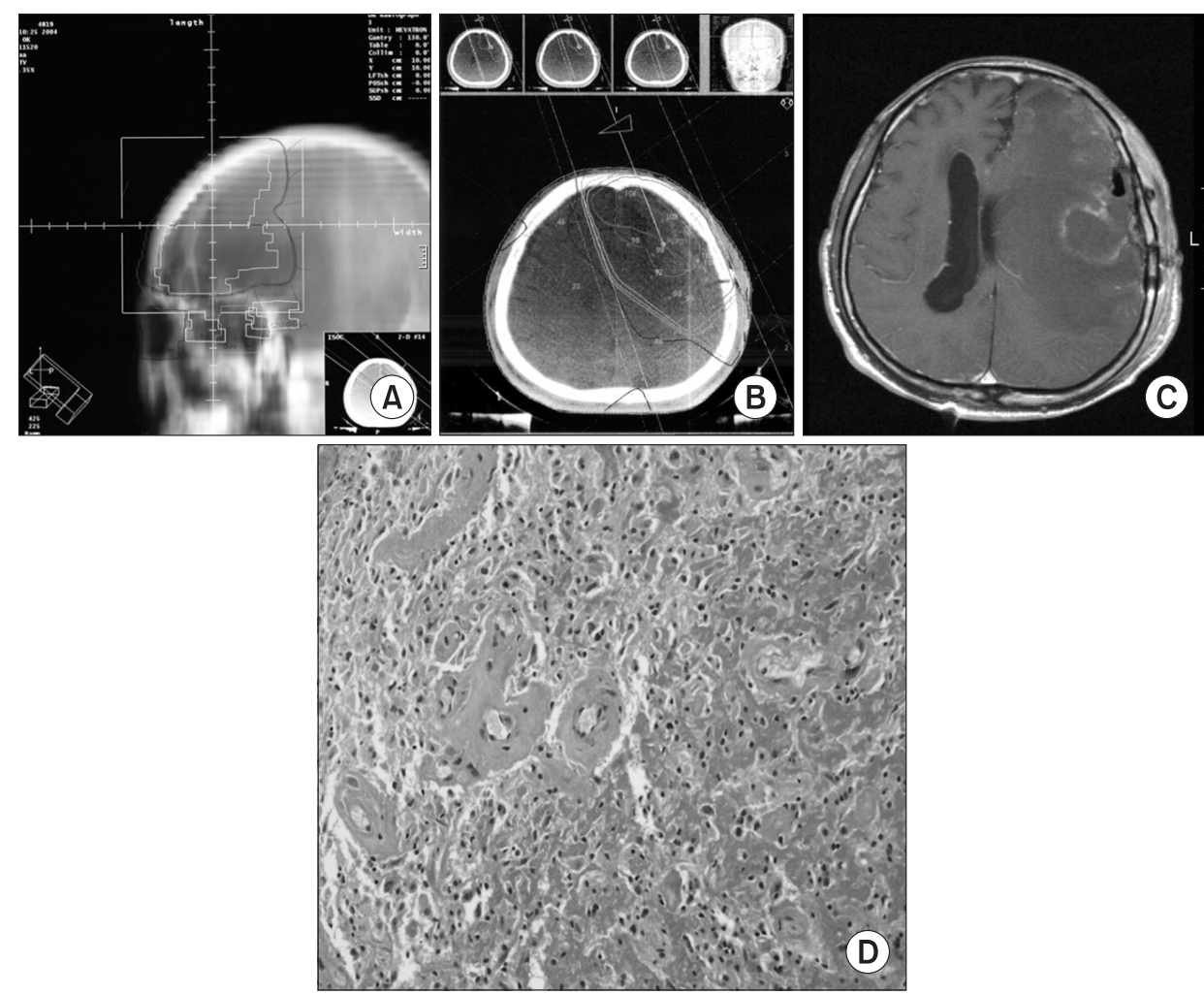

Fig. 2. A 59-year-old male (patient no. 5) diagnosed with glioblastoma multiform in the left frontal lobe following subtotal tumor resection and definitive radiotherapy with up to 59.4 Gy in 33 fractions over 45 days. The digitally reconstructed radiograph image $(A)$, isodose line distribution in treatment planning system (B), and thin gadolinium-enhancing lesion and surrounding edematous change with midline shifting on the T1weighted MR image taken 0.5 month after radiotherapy (C) are shown. He underwent re-operation, and histologic findings showed radiation-induced fibrinoid necrosis and prevascular collagen deposition (D) $\left(H \& E E_{1} \times 200\right)$. to compare the distribution of disease-associated factors between patients with RIBN and those with RIBC. However, we could not find any statistical differences among the variables. Gender $(p=0.061)$ and $\mathrm{BED}_{3}(p=0.084)$ were the only weak influencing factors. Six of the eight patients with RIBN were female and all of four patients with RIBC were male. Although the $B D_{3}$ for patients with RIBN was higher than that for those with RIBC, this was due to the use of radiosurgery in patients with RIBN. There was no difference in patient age ( $p=$ 1.000), concurrent chemotherapy $(p=0.515)$, maximum tumor diameter $(p=1.000)$, estimated 3D tumor volume $(p=1.000)$, surgical extent $(p=0.394)$, pathologic diagnosis $(p=1.000)$, or history of other diseases ( $p=0.545$ ) between RIBN and RIBC groups.

Since the majority of cases were treated according to an old version of the radiotherapy treatment planning system, we could not perform a detailed dose-volume analysis. To compare treatment volumes among patients, we indirectly estimated the total volumes by multiplying the three longest diameters of the GTV. Nine patients treated with conventional fractionated radiotherapy were analyzed. The estimated volumes of the four patients with RIBC were 19.2, 44.6, 120.7, and $111.7 \mathrm{~cm}^{3}$, and those of the five patients with RIBN were $15,37.3,105,144.7$, and $139.8 \mathrm{~cm}^{3}$.

\section{Survival assessment}

At the time of this analysis, eight patients had died and four patients were alive. Median follow-up duration after the initial diagnosis was 104.6 months (range, 68 to 218 months). Overall survival times from the time of initial diagnosis and RIBI development were determined. The overall survival times of one patient who was treated with definitive radiosurgery and another patient who was diagnosed with SCLC were calculated from the first day of brain irradiation. The median overall survival times from initial diagnosis or RIBI development for all patients were 38.2 months (range, 6.9 to 218.2 months) and 21.5 months (range, 3.4 to 175.8 months), respectively. Median overall survival times from the initial diagnosis and RIBI among GBM patients were 16.8 months (range, 6.9 to 135.6 months) and 9.4 months (range, 3.4 to 124.0 months), respectively.

We assessed the progression time to the RIBI by dividing the patients into two groups: less than 6 months (group A) and more than 6 months (group B). Although there was no statistical difference ( $p=0.318)$, PCV chemotherapy was predominantly used in group $A$. Five of six patients who were treated with concurrent PCV chemoradiation developed RIBI within 6 months. The overall survival time of group B was significantly longer than that of group A. Rank sum of group 
$A$ and $B$ was 24 and 54, respectively ( $p=0.016)$. We also performed a subgroup analysis of eight patients with high grade gliomas according to RIBI progression time. On the logrank test, group $B$ tended to have a higher overall survival rate compared to group $A(p=0.085)$. Kaplan-Meier overall survival curves are shown in Fig. 3 . Two (patient 5 and 7) among five patients who developed RIBI within 6 months were subsequently confirmed as disease recurrence in 6 and 14 months after $\mathrm{RIBI}$, respectively.

At the time of analysis, most of patients had recovered from major neurologic symptoms. However, four patients suffered from mild to moderate permanent neurologic sequelae.

\section{Discussion and Conclusion}

In 1950, Lowenberg-Scharenberg and Bassett [13] reported amyloid degeneration in human brain following $X$-ray therapy. Thereafter, multiple instances of cerebral radiation necrosis have been reported. It is difficult to assess the actual incidence of RIBN. This is because treatment sometimes includes combination of radiation and chemotherapy, and both of these treatment modalities are injurious to normal brain [14]. Moreover, it is difficult to differentiate between RIBN and the lesions related to recurrent brain tumors because RIBI mimics progressive or recurrent brain tumors. Pseudoprogression [15] has been defined as new areas of contrast enhancement with subsequent improvement without any further treatment of

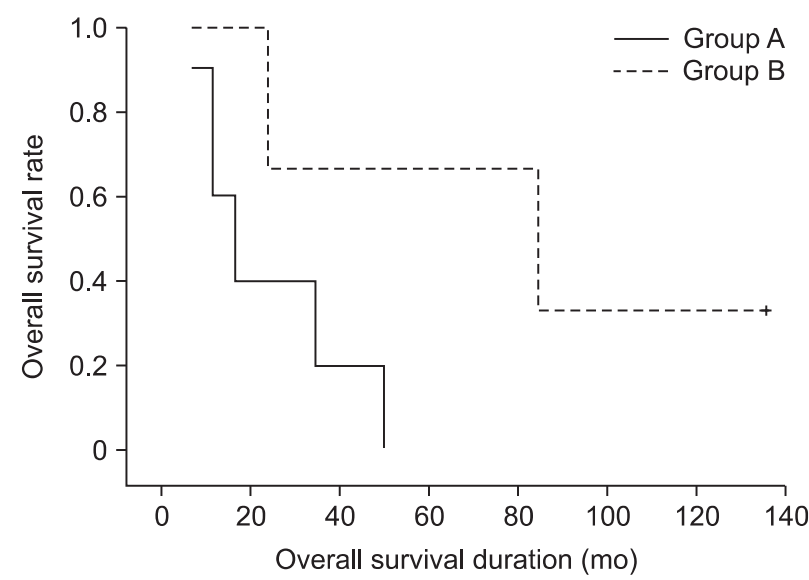

Fig. 3. Kaplan-Meier overall survival curves for eight patients with high grade gliomas are shown. Patients with radiationinduced brain injury (RIBI) within 6 months (group A) had lower survival rates compared to those who developed RIBI after 6 months (group $B)(p=0.085)$. Median overall survival times for group $A$ and $B$ were 16.8 and 84.6 months, respectively.
GBM after concurrent chemoradiation. Pseudoprogression is most likely to be induced by a pronounced local tissue reaction with an inflammatory component, edema, and abnormal vessel permeability causing new or increased contrast enhancement on neuroimaging. In severe cases, this condition can result in actual treatment-related necrosis. Although new diagnostic imaging techniques [16-18] made it possible to diagnose brain lesions more accurately, not all of the previous reports on RIBN involved histopathologically confirmed cases, which might implicate the underestimation of RIBN incidence.

In the current study, we reviewed 12 cases of histopathologically proven RIBI. Among these, eight cases were diagnosed with RIBN and the other four had different forms of radiation injury. All patients except for three had been treated with conventional fractionated radiation therapy. The total radiation doses were within the range tolerable for the normal brain. We investigated whether other risk factors influenced the development of RIBN. Although our study was limited due to it's small sample sizes, gender $(p=0.061)$ and $B D_{3}(p=$ 0.084) were the weak influencing factors. Among the patients who were treated with conventional fractionated radiation therapy, all of the RIBN cases occurred in patients treated with radiation therapy up to 59.4 Gy except for one patient. Although radiation dose, fraction size, and volume are wellknown factors affecting RIBN development $[19,20]$, age, gender and a history of conditions that affect the microvasculature, such as diabetes mellitus, hypertension, ischemic heart disease or brain infarction, could also be potential risk factors of RIBN [7]. In our study, six patients had these risk factors, and majority of the patients had more than one risk factor. Gender distribution showed similar results compared with a previous report [7]. Among the eight patients with RIBN, six were female. In contrast, all of four RIBC patients were male. $\mathrm{RIBI}$ after tolerable dose ranges of conventional fractionated radiotherapy is known to rarely develop, and concurrent use of chemotherapy might have contributed the development RIBI because eight among nine patients who were treated with conventional fractionated radiotherapy had received concurrent chemotherapy. In one study by Ruben et al. [14], the use of chemotherapy was strongly related to subsequent development of RIBN and the significance was maintained even after adjusting for BED.

In dose escalation study performed by Radiation Therapy Oncology Group [21], maximum tolerated doses in single fraction radiosurgery were $24 \mathrm{~Gy}, 18 \mathrm{~Gy}$, and $15 \mathrm{~Gy}$ for tumors $\leq 20 \mathrm{~mm}, 21-30 \mathrm{~mm}$, and 31-40 mm. In our study, doses and 
volumes of radiosurgery in all three patients were higher compared with those recommended in that trial. Moreover, one patient whose radiosurgery dose was $24 \mathrm{~Gy}$ had been previously treated with 24 Gy in 12 fractions in the whole brain as a prophylactic cranial irradiation, and another 24 Gy in 12 fractions to the whole brain after cancer recurrence within 22 months of total elapsed time for the entire radiotherapy course.

In the six GBM patients, three had relatively prolonged overall survival times (more than 2 years). All three patients had received concurrent chemotherapy. The overall survival times for the three prolonged survivors were 24.1, 84.6, and 135.6 months, respectively. The patient with an overall survival time of 135.6 months was still alive at the time of this analysis. We reconfirmed that her pathology was GBM which was characterized by a 40\% Ki-67 index, moderate cellularity, severe cellular pleomorphism, 36-40 mitotic activity, microvascular proliferation, and necrosis. Prolonged survival after a diagnosis of GBM is rare, and we noticed that the three long-term survivors were also diagnosed with RIBI. MGMT gene promoter methylation was revealed to be significantly correlated with development of pseudoprogression and improvement in survival [22]. We did not evaluate MGMT gene promoter methylation status in the present study, and we need to confirm this finding.

Our study may help define pseudoprogression [23] in cases of GBM . Since the interval between the end of radiotherapy and the diagnosis of brain necrosis was within 6 months in five patients, our results suggest that the progression found in follow-up imaging studies within this period could be regarded as pseudoprogressive lesions. Chamberlain et al. [24] reported similar findings in GBM patients. Twenty-six patients failed neuroradiographically within 6 months among 51 patients who had been treated concurrently with temozolomide and radiotherapy. Among 15 patients who underwent re-operation, seven patients were found to have necrosis without evidence of viable tumor cells. These concordant results suggest that pseudoprogression represents a continuum between subacute reaction to radiation and treatment-related necrosis. However, the presence of isolated and viable tumor cells could not be entirely excluded in the current study as well as the one by Chamberlain et al. [24].

Although pseudoprogression after concurrent chemoradiation with temozolomide $[23,24]$ in GBM patients is generally well-known, the role of PCV chemotherapy related to pseudoprogression is not well-defined. Although PCV chemotherapy for treating high grade gliomas is relatively inadequate for current trends, the feasibility and potency of PCV chemotherapy has been reported in previous literatures $[25,26]$. We observed early occurrences of RIBI in patients treated with PCV chemotherapy. However, patients who developed RIBI within 6 months had an inferior survival rate compared to those who developed RIBI after 6 months, and two among five patients who developed RIBI within 6 months subsequently showed disease progression. Even though various confounding factors could contribute the overall prognosis, we found that early RIBI still had potential risk of subsequent disease recurrence.

Although RIBN is a hazardous late brain injury associated with the potential risk of debilitating sequelae and lesion progression, it did not seem to negatively impact overall survival in our study. Moreover, we observed improved survival in the GBM patients despite suboptimal treatment. RIBN in GBM patients might have distinctive characteristics compared to other forms of brain necrosis in terms of early occurrence and prolonged survival. Further investigation to identify the underlying mechanism of pseudoprogression, prolonged survival in GBM patients, and the role of PCV chemotherapy as a potent radiosensitizer are required. Association between RIBI progression time and overall survival should be also examined in future studies.

Quality of life [27] is another concern for brain tumor patients due to the risk of permanent sequelae. Four patients in our study had suffered mild to moderate permanent sequelae. It is obvious that RIBN or RIBI is a serious late complication that most physicians wish to avoid. However, considering the limited life expectancy among patients with high grade tumors, optimal radiation treatment as well as aggressive surgical tumor removal should be offered.

Based on our study, we need to more accurately diagnose the progressive lesions in imaging studies after treatments, because discrimination of early recurrence in this period is essential for decision of further treatment strategy. Newer imaging techniques [16-18] may be helpful for noninvasive differential diagnosis. However, even in cases with suspicious of non-recurrent lesions, salvage operation should be considered in symptomatic patients with progressive neurologic deteriorations or functional changes.

Although we cannot draw any firm conclusions due to the small sample size of this study, we identified some consistent factors associated with RIBI. In our study, a history of diseases such as diabetes mellitus, hypertension, ischemic heart disease, 
or cerebral infarction; use of concurrent chemotherapy, gender, re-irradiation within a short period, and radiosurgery in a single high dose with large treatment volume seemed to be correlated with developing RIBI. However, this was not fully demonstrated in the statistical analysis. RIBN associated with conventional fractionated radiotherapy occurred in patients treated with a relatively lower dose range rather than established dose-volume parameters. GBM patients with RIBN showed prolonged overall survival despite the inclusion patients with suboptimal treatment schemes in our analysis.

In conclusion, our study showed that RIBN can occur within conventional dose range and shorter period in potentially high-risk group of patients. Special concern is required in these patients because of the potentially reduced quality of life associated with radiation injury. Overall survival in GBM patients who developed RIBI had prolonged overall survival time. However, RIBI that occurred within 6 months after PCV chemoradiation was associated with inferior overall survival compared to RIBI cases that developed after 6 months, probably due to the potential risk of subsequent disease progression in early period RIBI. More efficacious methods to discriminate true recurrence and $\mathrm{RIBI}$, and the relationship between RIBN and overall prognosis, especially in GBM patients should be further investigated in future large trials.

\section{Conflict of Interest}

No potential conflict of interest relevant to this article was reported.

\section{References}

1. Tsien C, Moughan J, Michalski JM, et al. Phase I threedimensional conformal radiation dose escalation study in newly diagnosed glioblastoma: Radiation Therapy Oncology Group Trial 98-03. Int J Radiat Oncol Biol Phys 2009;73:699708.

2. Andrews DW, Scott CB, Sperduto PW, et al. Whole brain radiation therapy with or without stereotactic radiosurgery boost for patients with one to three brain metastases: phase III results of the RTOG 9508 randomised trial. Lancet 2004;363:1665-72.

3. Floyd NS, Woo SY, I BS, et al. Hypofractionated intensitymodulated radiotherapy for primary glioblastoma multiforme. Int J Radiat Oncol Biol Phys 2004;58:721-6.

4. Roberge D, Petrecca K, El Refae M, Souhami L. Whole-brain radiotherapy and tumor bed radiosurgery following resection of solitary brain metastases. J Neurooncol 2009;95:95-9.

5. Bese NS, Uzel O, Turkan S, Okkan S. Continuous hyperfractionated accelerated radiotherapy in the treatment of highgrade astrocytomas. Radiother Oncol 1998;47:197-200.

6. Emami B, Lyman J, Brown A, et al. Tolerance of normal tissue to therapeutic irradiation. Int J Radiat Oncol Biol Phys 1991;21:109-22.

7. Lawrence $Y R$, Li XA, el Naqa I, et al. Radiation dose-volume effects in the brain. Int J Radiat Oncol Biol Phys 2010;76(3 Suppl):S20-7.

8. Langley RE, Bump EA, Quartuccio SG, Medeiros D, Braunhut SJ. Radiation-induced apoptosis in microvascular endothelial cells. Br J Cancer 1997;75:666-72.

9. Lyubimova N, Hopewell JW. Experimental evidence to support the hypothesis that damage to vascular endothelium plays the primary role in the development of late radiation-induced CNS injury. Br J Radiol 2004;77:488-92.

10. Nguyen $V$, Gaber MW, Sontag MR, Kiani MF. Late effects of ionizing radiation on the microvascular networks in normal tissue. Radiat Res 2000;154:531-6.

11. Li YQ, Jay V, Wong CS. Oligodendrocytes in the adult rat spinal cord undergo radiation-induced apoptosis. Cancer Res 1996;56:5417-22.

12. Chow BM, Li YQ, Wong CS. Radiation-induced apoptosis in the adult central nervous system is p53-dependent. Cell Death Differ 2000;7:712-20.

13. Lowenberg-Scharenberg K, Bassett RC. Amyloid degeneration of the human brain following X-ray therapy. J Neuropathol Exp Neurol 1950;9:93-102.

14. Ruben JD, Dally M, Bailey $M$, et al. Cerebral radiation necrosis: incidence, outcomes, and risk factors with emphasis on radiation parameters and chemotherapy. Int J Radiat Oncol Biol Phys 2006;65:499-508.

15. Brandsma D, Stalpers L, Taal W, Sminia P, van den Bent MJ. Clinical features, mechanisms, and management of pseudoprogression in malignant gliomas. Lancet Oncol 2008;9:453-61.

16. Ullrich RT, Kracht L, Brunn A, et al. Methyl-L-11C-methionine PET as a diagnostic marker for malignant progression in patients with glioma. J Nucl Med 2009;50:1962-8.

17. Hlaihel C, Guilloton L, Guyotat J, Streichenberger N, Honnorat J, Cotton F. Predictive value of multimodality MRI using conventional, perfusion, and spectroscopy MR in anaplastic transformation of low-grade oligodendrogliomas. J Neurooncol 2010;97:73-80.

18. Price SJ. The role of advanced MR imaging in understanding brain tumour pathology. Br J Neurosurg 2007;21:562-75.

19. Lee $A W$, Kwong $D L$, Leung $S F$, et al. Factors affecting risk of symptomatic temporal lobe necrosis: significance of fractional dose and treatment time. Int J Radiat Oncol Biol Phys 
2002;53:75-85.

20. Chin LS, Ma L, DiBiase S. Radiation necrosis following gamma knife surgery: a case-controlled comparison of treatment parameters and long-term clinical follow up. J Neurosurg 2001;94:899-904.

21. Shaw $E$, Scott $C$, Souhami $L$, et al. Single dose radiosurgical treatment of recurrent previously irradiated primary brain tumors and brain metastases: final report of RTOG protocol 90-05. Int J Radiat Oncol Biol Phys 2000;47:291-8.

22. Brandes $A A$, Franceschi $E$, Tosoni $A$, et al. MGMT promoter methylation status can predict the incidence and outcome of pseudoprogression after concomitant radiochemotherapy in newly diagnosed glioblastoma patients. J Clin Oncol 2008;26:2192-7.

23. Brandsma $D$, van den Bent MJ. Pseudoprogression and pseudoresponse in the treatment of gliomas. Curr Opin Neurol 2009;22:633-8.

24. Chamberlain MC, Glantz MJ, Chalmers L, Van Horn A, Sloan AE. Early necrosis following concurrent Temodar and radiotherapy in patients with glioblastoma. J Neurooncol 2007;82:81-3.

25. Brandes $A A$, Nicolardi $L$, Tosoni $A$, et al. Survival following adjuvant PCV or temozolomide for anaplastic astrocytoma. Neuro Oncol 2006;8:253-60.

26. Yang SH, Hong YK, Yoon SC, et al. Radiotherapy plus concurrent and adjuvant procarbazine, lomustine, and vincristine chemotherapy for patients with malignant glioma. Oncol Rep 2007;17:1359-64.

27. Budrukkar $A$, Jalali $R$, Dutta $D$, et al. Prospective assessment of quality of life in adult patients with primary brain tumors in routine neurooncology practice. J Neurooncol 2009;95:413-9. 